\title{
Estimation of the Effective Permeability in Magnetorheological Fluids*
}

October 23, 1998

\author{
Tammy M. Simon ${ }^{1}$, F. Reitich ${ }^{2}$, M.R. Jolly ${ }^{3}$, K. Ito ${ }^{1}$, H.T. Banks ${ }^{1}$
}

\begin{abstract}
Magnetorheological (MR) fluids constitute examples of controllable ("smart") fluids, whose rheological properties vary in response to an applied magnetic field. These fluids typically consist of micron-sized, magnetizable particles dispersed in a nonpermeable carrier fluid. The essential characteristic of MR fluids is that they may be continuously and reversibly varied from a state of free flowing liquids in the absence of an applied magnetic field to that of stiff semi-solids in a moderate field. Understanding the magnetic properties of MR fluids is crucial to the design of MR fluid-based devices and it also provides valuable insight into the character of the microstructure responsible for their field-dependent rheology. Prediction of the overall magnetic properties of MR composites is a challenging task, however, due to the highly nonlinear and oscillatory nature of the magnetization of the constituents. In this paper we propose a model for this behavior that is based on the mathematical theory of homogenization. We derive effective equations that govern the magnetic response of (periodically arranged) particle-chains through magnetic saturation. Comparison of numerical results for these equations with experimental data show good agreement which suggests that our approach could be useful in the design of improved MR fluids.
\end{abstract}

* Research supported in part by a US Department of Education Graduate Assistance in Areas of National Need (GAANN) Fellowship (TMS), the U.S. Air Force Office of Scientific Research under grants AFOSR F49620-95-1-0236 (HTB and KI), AFOSR F49620-98-1-0180 (HTB), AFOSR F49620-95-1-0375 (TMS), AFOSR F49620-95-1-0113 (FR), NSF DMS-9622555 (FR).

${ }^{1}$ Center for Research in Scientific Computations, Department of Mathematics, North Carolina State University Raleigh, NC 27695

${ }^{2}$ School of Mathematics, University of Minnesota, Minneapolis, MN 55455

${ }^{3}$ Advanced Technologies Research Group, Lord Corporation, Thomas Lord Research Center Cary, NC 27511-7900 


\section{Introduction}

Magnetorheological (MR) and electrorheological (ER) fluids constitute examples of controllable ("smart") fluids, whose rheological properties vary in response to an applied magnetic or electric field respectively. These fluids typically consist of micron-sized, polarizable particles dispersed in a non-permeable medium. The essential characteristic of MR (respectively ER) fluids is that they may be continuously and reversibly varied from a state of free flowing liquids in the absence of an applied magnetic (respectively electric) field to that of stiff semi-solids in a moderate field. This feature has inspired the design of a large variety of devices based on the use of MR and ER fluids, such as semi-active dampers, clutches, brakes, hydraulic valves and robotic control systems of various sorts [5, 9] (see also http://www.mrfluid.com).

Historically, both experimental and analytical research programs have primarily concentrated on ER fluids (see e.g. [8, 9] and the references therein). However, magnetorheological fluids have now been recognized to offer several advantages over their electric counterparts, including higher achievable yield stresses, a wider range of possible liquid carriers and lower sensitivity to impurities [5]. Prediction of their overall magnetic behavior, on the other hand, is a challenging task due to the highly nonlinear and oscillatory nature of the magnetization of the constituents. As stated, the characteristic size of the particles is several orders of magnitude smaller than that of a sample, rendering standard finite element modeling impractical.

In this paper we offer a model for MR fluid behavior that is based on the mathematical theory of homogenization. We obtain "effective" equations that govern the magnetic response of periodically arranged particle-chains through magnetic saturation. These "effective" or homogenized equations are obtained through a subtle limiting process that identifies the composite with a suitable homogeneous material as the particle size decreases (at fixed volume fractions). We do this in both the magnetically linear (near zero field) and nonlinear (through saturation) regimes. In both cases we numerically solve the resulting equations to obtain magnetization curves. These exhibit substantial agreement with experimental data (from experiments carried out at Lord Corporation).

This paper is organized as follows. In $\S 2$, we derive the constitutive equations and describe the MR effect. In $\S 3$, we obtain analytical formulas for the linear effective magnetic permeabilities. We also discuss the corresponding numerical calculations and compare these, along with standard approximations and bounds found in the literature, with experimental data. Finally in $\S 4$, we derive analytical formulas for the nonlinear effective magnetization and present the corresponding numerical results.

\section{MR Effect and Magnetostatics}

Magnetorheological materials consist of micron size (typically 2 to $5 \mu \mathrm{m}$ ) magnetically permeable particles (e.g, iron particles) dispersed in a non-permeable medium. Upon application of a field, the induced polarization of the particles causes the formation of chain-like struc- 
tures (roughly aligned with the field) which, in turn, are responsible for the "MR effect": a dramatic change in the rheological properties of the mixture (see Figure 1 and Figure 2) $[2,11,13]$. In a steady or quasi-steady configuration (such as that of Figure 1(b)), the magnetic field $\vec{H}$ and the magnetic induction $\vec{B}$ within the specimen must satisfy

$$
\nabla \cdot \vec{B}=0, \text { and } \nabla \times \vec{H}=\vec{J}
$$

where $\vec{J}$ denotes the free current. For the MR materials we consider here, $\vec{J} \equiv 0$ and thus we may write

$$
\vec{H}=-\nabla \Phi
$$

for a scalar potential $\Phi$. Moreover, the constituents in our MR fluids will be assumed isotropic and, therefore,

$$
\vec{B}=\vec{B}_{\mathbf{i}}+\mu_{0} \vec{H}=\mu \mu_{0} \vec{H}
$$

where $\vec{B}_{\mathrm{i}}$ is the intrinsic induction (i.e., the field due to magnetization), $\mu_{0}$ the permeability of vacuum and $\mu=\hat{\mu} I$ denotes the relative permeability of a material. Here $I$ denotes the $n \times n$ identity matrix where $n=2$ or $n=3$ depending on the geometry that we consider. In our case we have

$$
\hat{\mu}= \begin{cases}\mu_{\mathrm{p}} & \text { in the particles, } \\ \mu_{\mathrm{c}} & \text { in the carrier liquid. }\end{cases}
$$

Hence (1)-(3) reduces to the fundamental equation of magnetostatics

$$
\nabla \cdot(\mu \nabla \Phi)=0 \quad \text { in } \Omega
$$

where $\Omega \subset \mathbb{R}^{\mathrm{n}}(\mathrm{n}=2$ or $\mathrm{n}=3)$ denotes the region in space occupied by the sample.

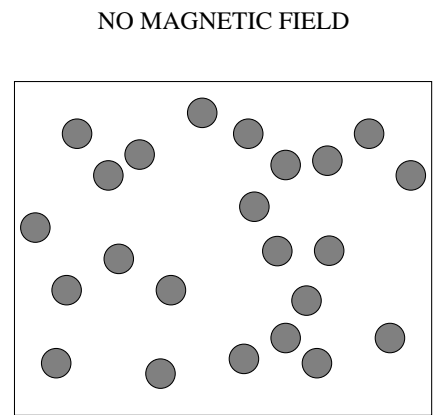

(a)

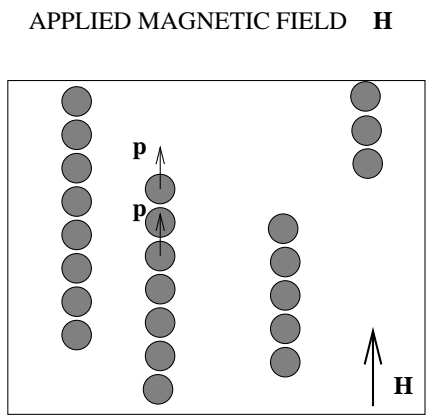

(b)

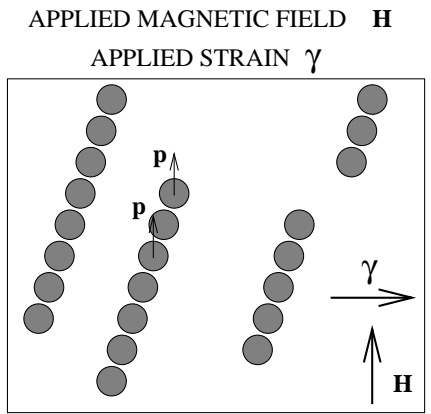

(c)

Figure 1: The MR effect: (a) the particles flow in the absence of magnetic fields; (b) they magnetize and form columns when a magnetic field is applied, thereby (c) changing and, indeed, dramatically increasing, the resistance to an applied shear strain.

To introduce the physically relevant boundary conditions that complement equation (5) it is important to consider a typical experimental arrangement as utilized in the measurement 


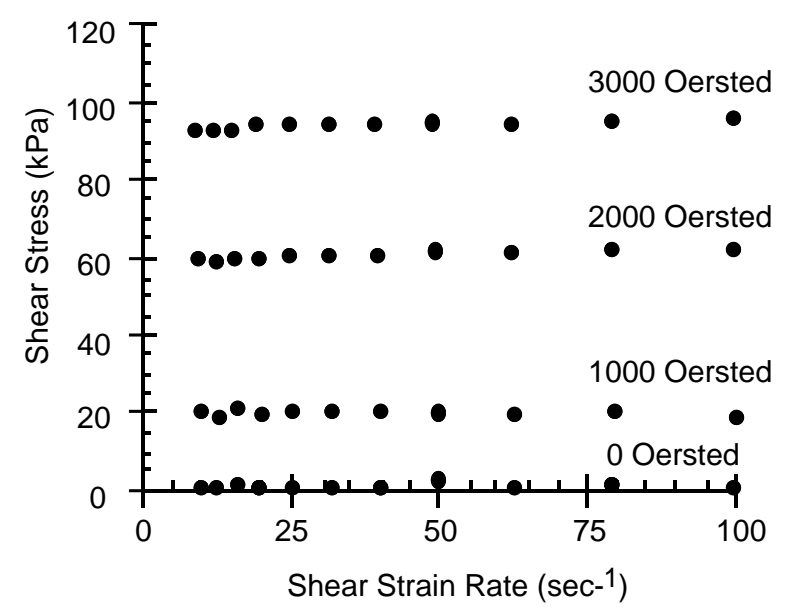

Figure 2: Shear strain rates versus shear stresses in the post-yield regime, for various values of the magnetic field intensity [11].

of the magnetic response of MR fluids. This arrangement (KJS model HG-500 magnetic Hysteresisgraph) is schematically depicted in Figure 3: a current is passed through an electromagnet and a probe is used to measure and control the applied field $\vec{H}$ where $\vec{H}=H_{3} \hat{k}$; the magnetic induction $\vec{B}=\vec{B}(\vec{H})$ can then be evaluated from the cylindrical sample with the aid of a search coil. Since the tangential components of $\vec{H}$ and the normal component

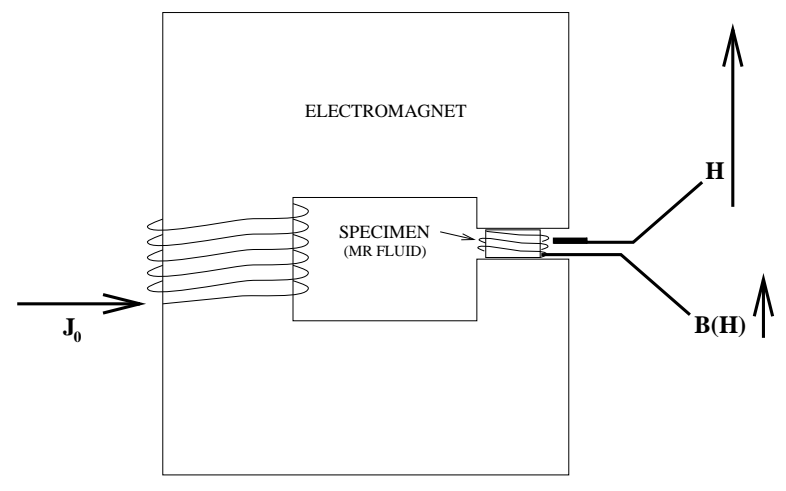

Figure 3: The experimental setup.

of $\vec{B}$ must be continuous across interfaces, it follows that

$$
H_{1}=H_{2}=0 \text { at the (flat) top } \mathcal{T} \text { and bottom } \mathcal{B}
$$

and

$$
\vec{B} \cdot \vec{n}=0 \text { at the (curved) sides } \mathcal{S} \text { of the specimen }
$$

where $\vec{n}$ is the unit normal vector to the surface $\mathcal{S}$ and, of course, $\partial \Omega=\mathcal{T} \cup \mathcal{B} \cup \mathcal{S}$. Results from such experiments are shown in Figure 4 for fluid samples with particle loadings of $10 \%$, 
$20 \%$, and $30 \%$ by volume. In each case, a single curve emerges as magnetic hysteresis was found to be negligible. Indeed, MR fluids are best described as superparamagnetic.

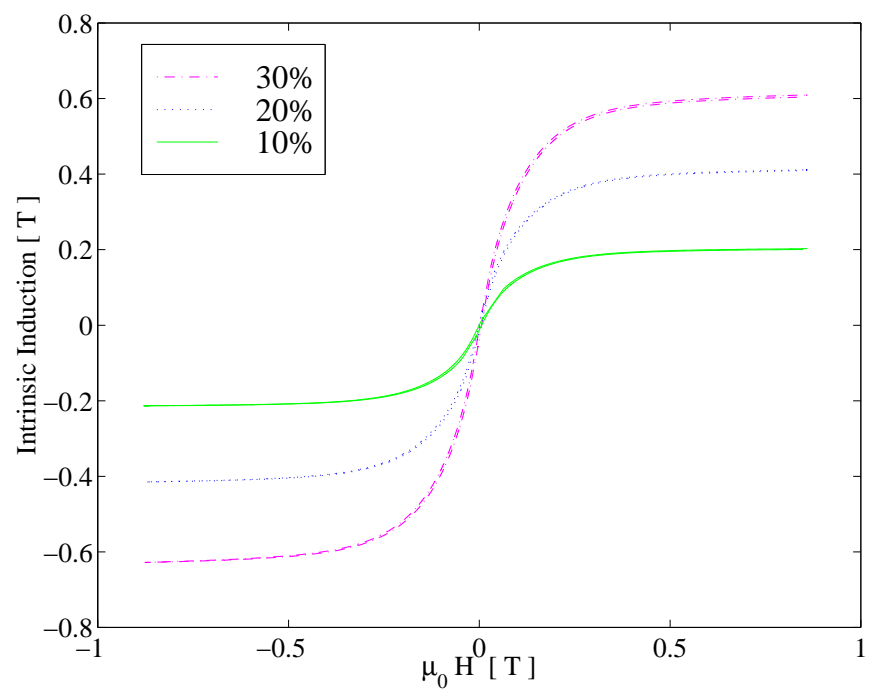

Figure 4: Experimental magnetic induction curves for three MR fluids of different iron volume percents.

\section{$3 \quad$ Linear Effective Permeability}

In the limit of low applied fields, the relation (3) can be taken to be linear; that is, $\mu$ is independent of the field itself. This quantity however remains highly oscillatory (see (4)). From the discussion in $\S 2$ (see Figure $1(\mathrm{~b})$ ), it seems natural to consider periodic structures. With this in mind, let $Y$ be the parallelogram defined by

$$
Y=\left[\frac{-c_{1}}{2}, \frac{c_{1}}{2}\right] \times \cdots \times\left[\frac{-c_{n}}{2}, \frac{c_{n}}{2}\right]
$$

for some constants $c_{i}$, and assume that $\mu$ is Y-periodic; that is, $\mu(x)=\mu\left(x+c_{i} e_{i}\right)$ for every $x \in \mathbb{R}^{\mathrm{n}}$ and for every $i=1, \ldots, n$. Here the vectors $e_{1}, \ldots, e_{n}$ are the usual unit direction vectors in $\mathbb{R}^{\mathrm{n}}$. Then, equation (5) can be written in the form (see [6])

$$
\nabla \cdot\left(\mu\left(\frac{x}{\epsilon}\right) \nabla \Phi^{\epsilon}\right)=0
$$

where $\mu=\mu(y)$ takes values in the periodicity cell Y given by (4) and $\epsilon$ is proportional to the (small) size of the particles (see Figure 5). The boundary conditions are the same as described in (6)-(7). Rather than seek a direct treatment of the governing equations, we study the system asymptotically as $\epsilon \rightarrow 0$ with the goal of replacing the heterogeneous material with a suitable homogeneous material whose macroscopic magnetic properties closely resemble those of the heterogeneous material. It is to be noted however, that this limiting process entails 

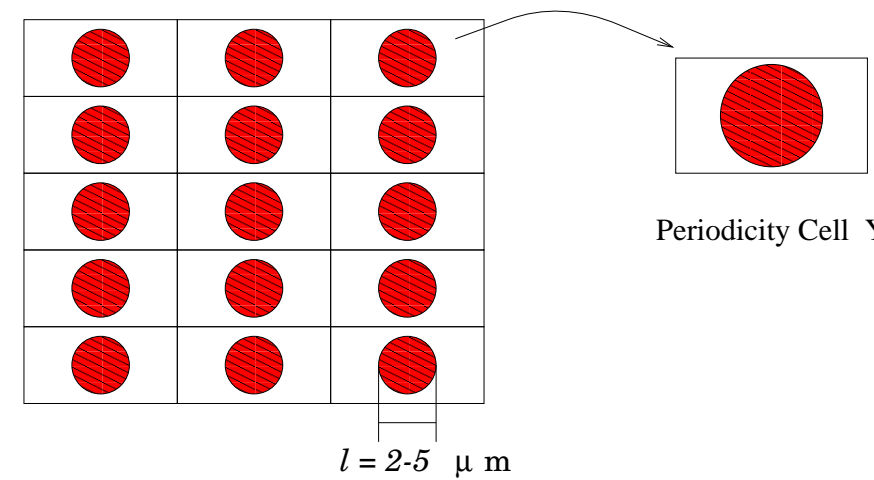

Periodicity Cell Y

Figure 5: Schematic of the periodic microgeometry assumed here.

rather subtle mathematical issues as is exemplified by the fact that the highly oscillating local fields $\left\{\nabla \Phi^{\epsilon}\right\}$ cannot be expected to converge pointwise in the sample but rather on "average". These issues of convergence (particularly weak convergence) are treated rigorously by the mathematical theory of homogenization. We recall for the reader that in a general Hilbert space $H$ with inner product $\langle$,$\rangle , we say f_{n}$ converges weakly to f (written $f_{n} \rightarrow f$ ) if $\left\langle f_{n}, g\right\rangle \rightarrow\left\langle f_{n}, g\right\rangle$ for all $g \in H$. With this in mind, we describe the basic ideas below.

\subsection{Homogenization}

We begin by considering the general Dirichlet boundary value problem

$$
\left\{\begin{array}{l}
-\nabla \cdot\left(\mu\left(\frac{x}{\epsilon}\right) \nabla \Phi^{\epsilon}\right)=f \text { on } \Omega \\
\Phi^{\epsilon} \in H_{0}^{1}(\Omega)
\end{array}\right.
$$

where $\Omega \subset \mathbb{R}^{\mathrm{n}}$ is considered a piece of heterogeneous material and

$$
\begin{aligned}
& H^{1}(\Omega)=\left\{u \in L^{2}(\Omega), \nabla u \in L^{2}\left(\Omega, \mathbb{R}^{\mathrm{n}}\right)\right\}, \\
& H_{0}^{1}(\Omega)=\left\{u \in H^{1}(\Omega), u=0 \text { on } \partial \Omega\right\} .
\end{aligned}
$$

We assume that $\mu(x)=\left(\mu_{i j}(x)\right), i, j=1, \ldots, n$ is Y-periodic, symmetric positive definite, and each $\mu_{i j}$ is bounded. With these conditions, we study the behavior of the system (9) in the limit as $\epsilon \rightarrow 0$. One can show (see [6]) that the solutions $\Phi^{\epsilon}$ to $(9)$ converge to the solution $\Phi^{0}$ of the following effective or homogenized equation

$$
\left\{\begin{array}{l}
-\nabla \cdot\left(\bar{\mu}_{\mathrm{hom}} \nabla \Phi^{0}\right)=f \text { on } \Omega \\
\Phi^{0} \in H_{0}^{1}(\Omega) .
\end{array}\right.
$$

The constant matrix $\bar{\mu}_{\text {hom }}$ is defined (see, e.g., [6]) by

$$
\left(\bar{\mu}_{\mathrm{hom}}\right)_{i k}=\frac{1}{|Y|} \int_{Y}\left(\mu_{i k}(y)+\sum_{j=1}^{n} \mu_{i j}(y) \frac{\partial \omega_{k}}{\partial y_{j}}\right) d y .
$$


where $\omega_{k}$ is the unique solution to the cell problem

$$
\left\{\begin{array}{l}
\nabla \cdot\left(\mu(y)\left(e_{k}+\nabla \omega_{k}(y)\right)\right)=0 \text { in } Y \\
\omega_{k} \in H_{\#}^{1}(Y) .
\end{array}\right.
$$

Here $H_{\#}^{1}(Y)$ denotes the set of periodic functions in $H^{1}(Y)$. The constant matrix $\bar{\mu}_{\mathrm{hom}}$ then represents the permeability of the "homogenized material" which, from a macroscopic view, approximates the permeability of the heterogeneous material with the given periodic microstructure. Note that although (12) cannot, in general, be explicitly solved, solutions to these non-oscillatory equations can be easily approximated by standard finite element methods. Moreover, if $\mu(y)$ is even in $y_{k}$ for all $k$ and the constituents are isotropic, then $\bar{\mu}_{\text {hom }}$ is a diagonal matrix and $\omega_{k}$ is odd in $y_{k}$ and even in $y_{j}$, for $j \neq k$. Then, from this and the periodicity of $\omega_{k}$, it follows that the periodic problem (12) reduces (e.g., see [1]) to the boundary value problem on the smaller domain $\tilde{Y}=\left[0, \frac{c_{1}}{2}\right] \times \cdots \times\left[0, \frac{c_{n}}{2}\right]$ :

$$
\begin{aligned}
\nabla \cdot\left(\mu ( y ) \left(e_{k}\right.\right. & \left.+\nabla \omega_{k}(y)\right)=0 \\
\omega_{k} & =0 \text { on } Y_{1}^{k} \\
\frac{\partial \omega_{k}}{\partial n} & =0 \text { on } Y_{2}^{k}
\end{aligned}
$$

where

$$
\begin{aligned}
Y_{1}^{k} & =\left\{y \in \partial \tilde{Y} \mid y_{k}=0, c_{k} / 2\right\}, \\
Y_{2}^{k} & =\partial \tilde{Y}-Y_{1}^{k} .
\end{aligned}
$$

Then, the effective permeability is obtained by numerically solving the boundary value problems (13) and substituting into the relation

$$
\left(\bar{\mu}_{\mathrm{hom}}\right)_{k k}=\frac{2^{n}}{|Y|} \int_{\tilde{Y}} \mu_{k k}(y)\left(1+\frac{\partial \omega_{k}}{\partial y_{k}}\right) d y
$$

\subsection{Cubic arrangements: numerical experiments, approximations and bounds}

We now turn to a discussion of how the numerical results for the problem discussed in $\S 3.1$ compare with other numerical approximations and bounds found in the literature. To do this, the specific geometry that we consider consists of a three-dimensional static configuration of spherical iron inclusions with permeability $\mu_{p}=2000$ uniformly dispersed (on a cubic lattice) in a carrier fluid with permeability $\mu_{c}=1$. We remark that we treat more realistic geometries, such as chain-like structures, in the following section. However, we consider this case as a means of comparison with other established estimates. For simplicity, it is assumed that all lengths are dimensionless and the basic periodicity cell is the unit cube in $\mathbb{R}^{3}$.

In addition to equation (14), other estimates for the effective properties of heterogeneous materials can be found in the literature. Perhaps the best known estimate for the effective 
properties of two-phase composites is attributed to Maxwell [10, 16, 18]. In this relation, it is assumed that the particles are far enough apart that the field induced by each particle only depends on the applied field and not on that of the other particles. Maxwell's estimate is given by

$$
\bar{\mu}_{\mathrm{M}}=\mu_{\mathrm{c}}\left(\frac{\mu_{\mathrm{p}}+2 \mu_{\mathrm{c}}+2 \phi\left(\mu_{\mathrm{p}}-\mu_{\mathrm{c}}\right)}{\mu_{\mathrm{p}}+2 \mu_{\mathrm{c}}-\phi\left(\mu_{\mathrm{p}}-\mu_{\mathrm{c}}\right)}\right)
$$

where $\phi=\frac{4}{3} \pi r^{3}$ denotes the volume fraction of the inclusions and $r$ represents a dimensionless radius (that is, a ratio of lengths).

More advanced estimates can be found in the literature. One such example is based on work by Rayleigh. Taking into account recent extensions of Rayleigh's work, namely by Lam $[15,18]$, we have

$$
\bar{\mu}_{\mathrm{R}}=\mu_{\mathrm{c}}\left(1-3 \phi \alpha^{(1)} / \Lambda\right),
$$

where

$$
\begin{gathered}
\Lambda=1+\alpha^{(1)} \phi-1.3047 \alpha^{(1)} \alpha^{(3)} \phi^{10 / 3} \\
-0.072317 \alpha^{(1)} \alpha^{(5)} \phi^{14 / 3} \\
-0.52894 \alpha^{(1)} \alpha^{(3)} \phi^{17 / 3} \\
-0.15256 \alpha^{(1)} \alpha^{(7)} \phi^{6}
\end{gathered}
$$

and

$$
\alpha^{(n)}=\frac{n\left(\mu_{c}-\mu_{p}\right)}{n\left(\mu_{c}+\mu_{p}\right)+\mu_{c}} .
$$

Because the Maxwell and Rayleigh-Lam estimates are based on asymptotic analysis (they are exact only as $\phi \rightarrow 0$ ), they do not accurately predict the behavior of the effective permeability as the particles begin to touch (that is, as $\phi \rightarrow \pi / 6$ ). For the simple cubic lattice of hard inclusions (e.g., infinitely permeable spheres), J.B. Keller found that the singularity at $\phi=\pi / 6$ is approximately logarithmic [14, 18]. Based on this work. Doyle suggested the interpolation formula [7, 18]

$$
\bar{\mu}_{d}=\mu_{c}\left[1+\frac{\pi}{2} \log \left(1-\frac{6 \phi}{\pi}\right)\right] .
$$

In addition to the estimates above, one can also derive bounds for the effective permeability $\mu_{\mathrm{eff}}$. The most general bounds are those that do not depend on the particular microgeometrical arrangement of the components. Among these we encounter, for instance, the so-called Hashin-Shtrikman bounds which, in our case, are given by

$$
\mu_{c}\left(1+\frac{3 \phi\left(\mu_{p}-\mu_{c}\right)}{3 \mu_{c}+(1-\phi)\left(\mu_{p}-\mu_{c}\right)}\right) \leq \mu_{\mathrm{eff}} \leq \mu_{p}\left(1-\frac{3(1-\phi)\left(\mu_{p}-\mu_{c}\right)}{3 \mu_{p}-\phi\left(\mu_{p}-\mu_{c}\right)}\right) .
$$

In Figure 6 we compare the numerical results obtained by linear homogenization for the vertical component of the effective permeability with results from equations (15)-(20). The homogenization estimates were obtained by numerically solving equations (13)-(14) using a commercial finite-element software package [17]. All of the estimates are in good agreement up to about $30 \%$ volume fraction, while only the Doyle estimates agree with the homogenization results throughout the entire range. 


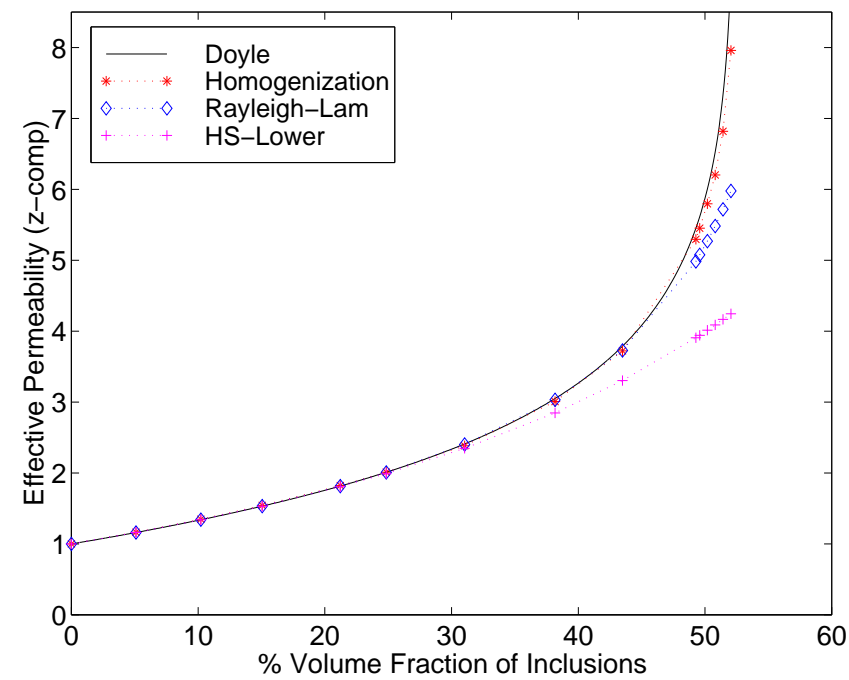

Figure 6: Effective permeability of two-phase composites with uniformly dispersed spherical inclusions. Here, the Maxwell estimate coincides with the Hashin-Shtrikman lower bound.

\subsection{Particle chains: numerical results vs experimental data}

In this section we consider a more realistic geometry including chain-like clusters and compare the homogenization estimates with experimental data. We note that the estimates of Maxwell, Rayleigh-Lam and Doyle are not applicable in this case since the particles are not dispersed uniformly throughout the fluid. The specific geometry that we now consider consists of a three-dimensional static configuration of spherical iron inclusions that form single chains. On each chain, the particles are separated by a fixed distance, $2 \alpha$ (see Figure 7 ), and the chains are periodically dispersed throughout the carrier fluid. This interparticle distance has a physical interpretation: due to processing procedures in construction of the fluids, the iron particles have an oxide outer layer that prevents the particles from actually touching when they are densely packed along the chains. Based on our approximation of the order of the thickness of the oxide layer (on the order of a nanometer for particles with radius on the order of a micron) the ratio of the interparticle distance to the particle diameter, denoted by $a$, is on the order of $10^{-2}-10^{-3}$. We take the (fixed) period along the direction of the applied field to be unity, and the period in the other directions to be $l$. Then, the periodic microstructure is characterized by the periodicity cell $Y=[-l / 2, l / 2]^{2} \times[-1 / 2,1 / 2]$ as is shown in Figure 7 . The slope of the experimental $\mathrm{B}-\mathrm{H}$ curve (see Figure 4 ) near $\mathrm{H}=0$ provides the permeability of each sample in the linear regime. We calculated this slope using the method of least squares. The linear regime was chosen as the interval corresponding to the fit that yielded the minimum mean-squared deviation. In Figure 8, the experimental data are shown along with the results of our homogenization calculations based on formulas (13)-(14) for values of the ratio $a=.0005, .001, .002, .004$. We find the agreement to be remarkable, given the highly idealized nature of the assumed microstructure. Indeed, some degree of polydispersity was certainly present within our samples and, of course, the 


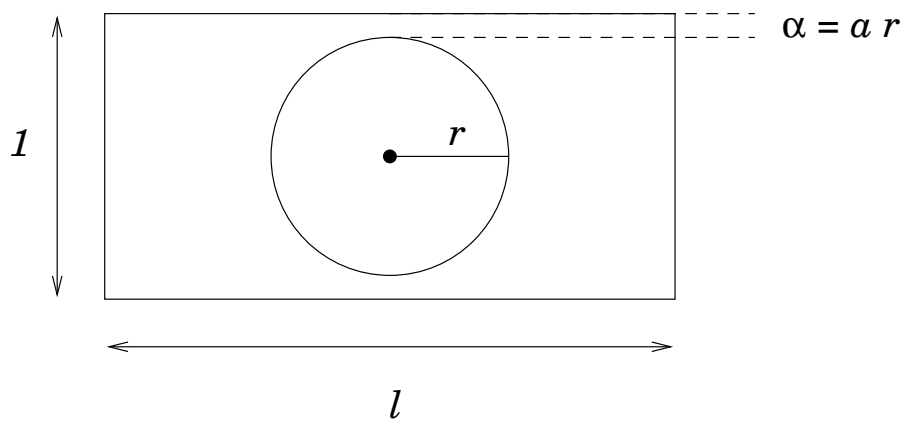

Figure 7: Cross section of the 3D periodicity cell.

true microgeometrical arrangements were more complex than simple linear chains. Indeed. this complexity is accentuated at larger volume fractions which may explain the slight increase in the deviation of our computed results from the experimental data as the particle concentration is increased.

The results above suggest that linear effective medium theories may provide us with a means to investigate the magnetic properties of MR fluids at low field intensities. At moderate fields however, magnetic saturation dramatically changes the particles' response. Interestingly, as we show in the next section, the main ideas of the theory of homogenization can be carried over to deal with nonlinear problems.

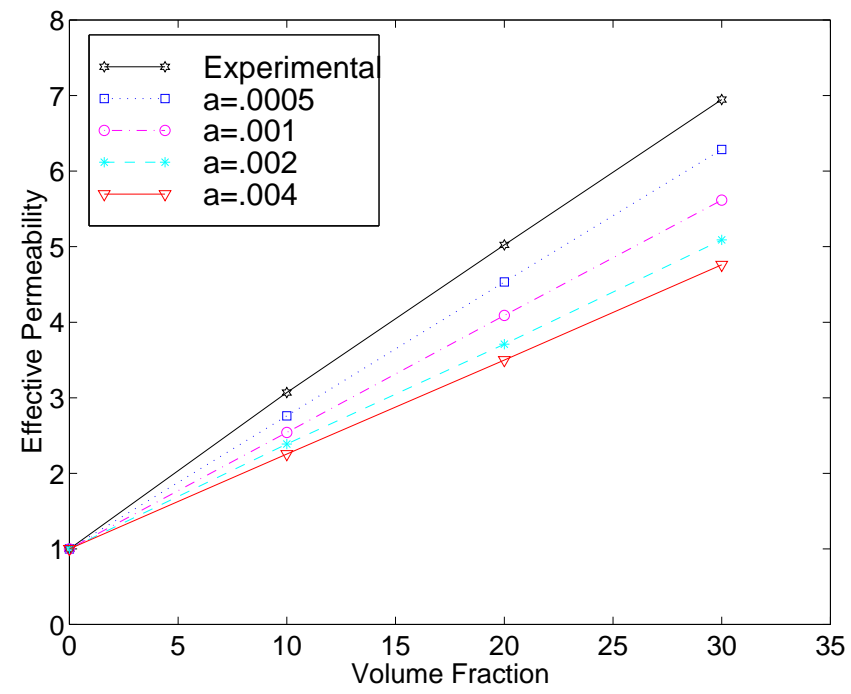

Figure 8: The $\epsilon_{3}$-component of the effective permeability of two-phase composites with periodic particle-chain structure. Numerical estimates for different values of $a$ are compared to experimental data. 


\section{Nonlinear Effective Magnetization}

As suggested by Figure 4, magnetic materials such as iron do possess, in general, a maximum attainable magnetization $B_{s}$ that, once achieved, does not allow any further magnetization. This statement implies that the relative permeability $\mu$ is, in fact, a function of the magnetic field $H$ (or equivalently of the magnetic induction $B$ ). Indeed, the relative permeability drops to a value near (and slightly above) one once a critical magnetic field $H_{\text {crit }}$ is exceeded. For instance, an empirical constitutive relation for the macroscopic permeability of iron in the presence of a magnetic field $\vec{H}$ can be derived from the Fröhlich-Kennelly relation [4]

$$
\mu=\mu(\vec{H})=1+\frac{\left(\mu_{\mathrm{p}}-1\right) B_{s}}{\mu_{0}\left(\mu_{\mathrm{p}}-1\right)|\vec{H}|+B_{s}},
$$

where $\mu_{\mathrm{p}}$ denotes the linear permeability of the inclusions. Thus, in general, problem (5)(7) involves the solution of a boundary value problem for highly oscillatory and nonlinear differential equations. As in the linear case, rather than seeking a direct solution of the governing equations, we employ the method of homogenization.

\subsection{Nonlinear Homogenization}

We begin by considering the general nonlinear Dirichlet boundary value problem

$$
\left\{\begin{array}{l}
-\nabla \cdot\left(b\left(\frac{x}{\epsilon}, \nabla \Phi^{\epsilon}\right)\right)=f \text { on } \Omega, f \in L^{2}(\Omega), \\
\Phi^{\epsilon} \in H_{0}^{1}(\Omega) .
\end{array}\right.
$$

In our case, $b(y, \xi)=\mu(y, \xi) \xi$. The limiting behavior of this system is characterized by the following result (for proof, see $[6]$ ):

Theorem 4.1 Assume that b(y, $\xi)$ in equation (2D) is measurable and periodic in $y$, Lipschitz continuous and strongly monotone in $\xi$, that is,

$$
\begin{gathered}
\left|b\left(y, \xi_{1}\right)-b\left(y, \xi_{2}\right)\right| \leq \alpha\left|\xi_{1}-\xi_{2}\right|, \\
\left(b\left(y, \xi_{1}\right)-b\left(y, \xi_{2}\right), \xi_{1}-\xi_{2}\right) \geq \beta\left|\xi_{1}-\xi_{2}\right|^{2} .
\end{gathered}
$$

Let $\left\{\Phi^{\epsilon}\right\}$ be the sequence of solutions to (2D). Then,

$$
\begin{aligned}
& \Phi^{\epsilon} \rightarrow \Phi^{0} \text { weakly in } H^{1}(\Omega), \\
& b\left(\frac{x}{\epsilon}, \nabla \Phi^{\epsilon}\right) \rightarrow b_{\mathrm{hom}}\left(\nabla \Phi^{0}\right) \text { weakly in } L^{2}\left(\Omega, \mathbb{R}^{\mathrm{n}}\right) .
\end{aligned}
$$

where $\Phi^{0}$ is the unique solution to the homogenized problem

$$
\left\{\begin{array}{l}
-\nabla \cdot\left(b_{\mathrm{hom}}\left(\nabla \Phi^{0}\right)\right)=f \text { on } \Omega \\
\Phi^{0} \in H_{0}^{1}(\Omega) .
\end{array}\right.
$$


The operator $b_{\mathrm{hom}}: \mathbb{R}^{\mathrm{n}} \rightarrow \mathbb{R}^{\mathrm{n}}$ is defined for every $\xi \in \mathbb{R}^{\mathrm{n}}$ by

$$
b_{\mathrm{hom}}(\xi)=\frac{1}{|Y|} \int_{Y} b\left(y, \xi+\nabla \omega^{\xi}(y)\right) d y,
$$

where $\omega^{\xi}$ is the unique solution to the local problem

$$
\left\{\begin{array}{l}
\int_{Y}\left(b\left(y, \xi+\nabla \omega^{\xi}(y)\right), \nabla v(y)\right) d y=0, \text { for every } v \in H_{\#}^{1}(Y), \\
\omega^{\xi} \in H_{\#}^{1}(Y) .
\end{array}\right.
$$

The most stringent condition in the above result is that of monotonicity. However, if the Fröhlich-Kennelly relation (21) is used to model the permeability of the iron particles, this condition is indeed satisfied. To see this, we consider the map $b(\vec{H})=\mu(\vec{H}) \vec{H}$ and show that it is monotone (see (24)). To do so, it suffices to show that the derivative of $b$ is positive definite. In $\mathbb{R}^{2}$, we set $\vec{H}=\left(H_{1}, H_{2}\right)$ and show that $\frac{\partial b_{1}}{\partial H_{1}}$ and the determinant of $\nabla b$ both are positive. Now

$$
\frac{\partial b_{1}}{\partial H_{1}}=1+\frac{\left(\mu_{p}-1\right) B_{s}}{\mu_{0}\left(\mu_{p}-1\right)|\vec{H}|+B_{s}}-\frac{\mu_{0} B_{s}\left(\mu_{p}-1\right)^{2} H_{1}^{2}}{\left[\mu_{0}\left(\mu_{p}-1\right)|\vec{H}|+B_{s}\right]^{2}|\vec{H}|}
$$

or setting $A=\mu_{0}\left(\mu_{p}-1\right)|\vec{H}|+B_{s}$,

$$
\frac{\partial b_{1}}{\partial H_{1}}=1+\frac{\left(\mu_{p}-1\right) B_{s}}{A^{2}}\left(A-\frac{H_{1}^{2} \mu_{0}\left(\mu_{p}-1\right)}{|\vec{H}|}\right) .
$$

Simplifying the term in the parenthesis, we have

$$
\frac{H_{2}^{2} \mu_{0}\left(\mu_{p}-1\right)+B_{s}|\vec{H}|}{|\vec{H}|}>0 \text { for all }\left(H_{1}, H_{2}\right) \neq(0,0),
$$

and hence, $\frac{\partial b_{1}}{\partial H_{1}}>0$. To see that the $\operatorname{det}(\nabla b)$ is positive as well, note that we may write

$$
\begin{aligned}
\operatorname{det}(\nabla b) & =\left(1+\alpha-\beta_{1}\right)\left(1+\alpha-\beta_{2}\right)-\beta_{1} \beta_{2} \\
& =(1-\alpha)\left[(1+\alpha)-\left(\beta_{1}+\beta_{2}\right)\right]
\end{aligned}
$$

where $\alpha=\left(\mu_{p}-1\right) B_{s} / A, \beta_{i}=H_{i}^{2}\left(\mu_{p}-1\right)^{2} B_{s} \mu_{0} /\left(A^{2}|\vec{H}|\right)(\mathrm{i}=1,2)$. Thus,

$$
\operatorname{det}(\nabla b)=(1+\alpha)\left[(1+\alpha)-\left(\beta_{1}+\beta_{2}\right)\right]
$$

where

$$
\beta_{1}+\beta_{2}=|\vec{H}|\left(\mu_{p}-1\right)^{2} B_{s} \mu_{0} / A^{2} .
$$

Finally, we have

$$
\begin{aligned}
1+\alpha & =1+\left(\mu_{p}-1\right) A B_{s} / A^{2} \\
& =1+\left(\mu_{p}-1\right)\left[\mu_{0}\left(\mu_{p}-1\right)|\vec{H}|+B_{s}\right] B_{s} / A^{2} \\
& =1+\left(\mu_{p}-1\right) B_{s}^{2} / A^{2}+|\vec{H}|\left(\mu_{p}-1\right)^{2} B_{s} \mu_{0} / A^{2} \\
& \geq \beta_{1}+\beta_{2}
\end{aligned}
$$


so that, from (32), it follows that $\operatorname{det}(\nabla b)>0$.

With regard to numerical issues, the computational challenges associated with the homogenization of nonlinear systems are significant. For linear materials, one only has to solve, at most, three (linear) boundary value problems of the form (12). However, a numerical evaluation of the effective permeability for nonlinear media (see (28)) is substantially more involved since this case requires solving a continuum of nonlinear problems. Next we present such results for a $2 \mathrm{D}$ geometry.

\subsection{D nonlinear numerical results vs experimental data}

For the nonlinear computations, we consider a two-dimensional configuration of circular particles that form single chains. As in $\S 3.3$, the particles along each chain are separated by a fixed distance denoted by $2 \alpha=2 a r$ (see Figure 7). Now the periodicity cell is taken to be $Y=[-l / 2, l / 2] \times[-1 / 2,1 / 2]$. We numerically solved the nonlinear elliptic equations (27)(28) using a standard finite elements program for a discrete set of points $\xi$ in $(0,0.8) \times(0,0.8)$. In this case, we can construct the effective B-H curve by the graphic interpolation of the discretely calculated values of $b_{\mathrm{hom}}(H)$ for $\xi=\left(0, \xi_{2}\right), \xi_{2} \in(0,0.8)$ where $\xi=\mu_{0} H$. The resulting $b_{\text {hom }}(H)$ (assuming the ratio $a=.002$ ) is compared with the experimental data in Figure 9. The vertical axis represents $B_{i}(H)=b_{\text {hom }}(H)-\mu_{0} H$, the field due to magnetization.

In order to investigate the dependence of $b_{\text {hom }}(H)$ on the ratio $a$ under our $2 \mathrm{D}$ model geometry, we computed the effective B-H curve for several values of $a$ between .002 and .02. As a result, we found that $b_{\text {hom }}(H)$ corresponding to $a=.016$ provides the best fit of the data for all three fluids from the linear regime through saturation. Data for the $30 \%$ sample is shown in Figure 10 (results are similar for the other two fluids).

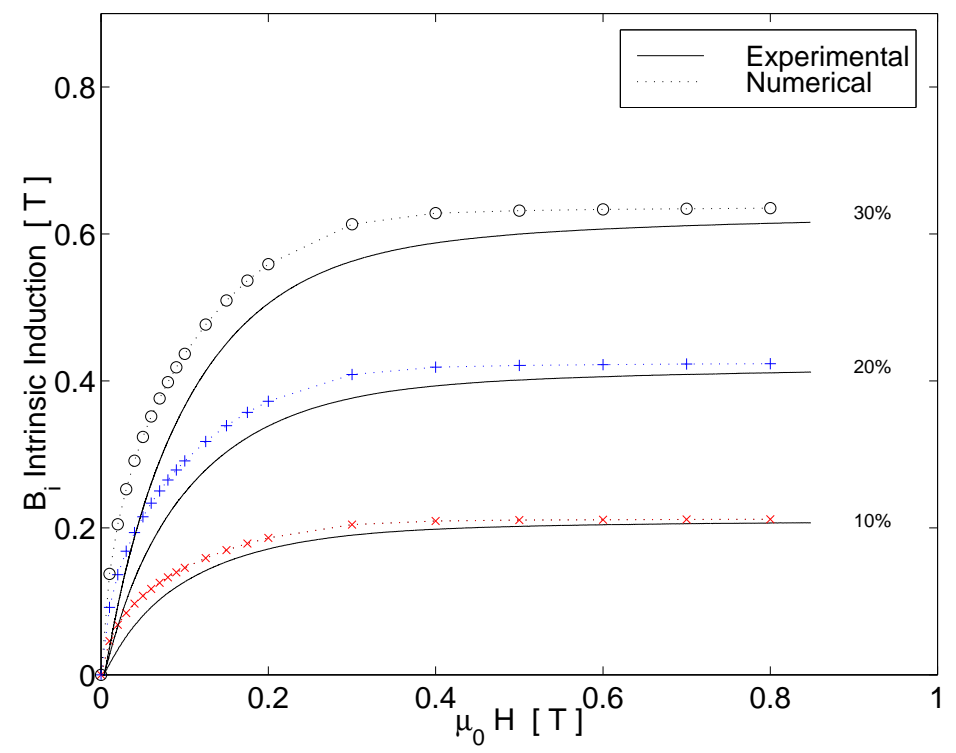

Figure 9: Effective magnetization as predicted by homogenization $(a=.002)$ compared to experimental data. 


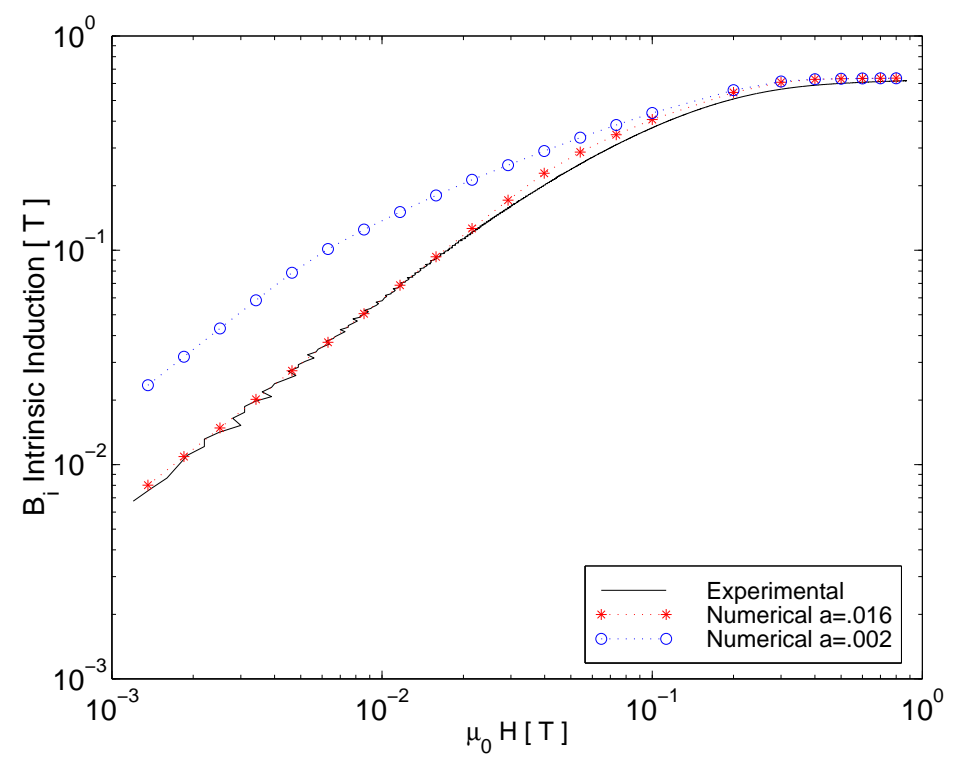

Figure 10: Effective magnetization computed for $a=.016, .002$ for the $30 \%$ sample.

\section{Conclusions}

A model for the effective magnetic properties of MR fluids was proposed that is based on the theory of homogenization. Analytical formulas for (periodic) linear and nonlinear media in both $\mathbb{R}^{2}$ and $\mathbb{R}^{3}$ were discussed. For the numerical results, we considered microstructures of monodispersed spherical inclusions arranged periodically in linear particle chains. Interestingly, even with this simplified model, the homogenization results predicted the experimental data with acceptable accuracy. However, the method is not restricted to these specific microstructures or particle shapes. Hence, homogenization provides an efficient way to estimate the magnetic properties of these fluids with reasonable accuracy without restricting the order, geometry or volume fraction of inclusions that may be considered.

\section{References}

[1] N. Bakhvalov and G. Panasenko, Homogenization: Averaging Processes in Periodic Media, Kluwer Academic, Boston (1989).

[2] C. P. Bean and I. S. Jacobs, Magnetization of a dilute suspension of a multidomain ferromagnetic, J. Appl. Phys. 31 (1960), 1228-1230.

[3] A. Bensoussan, J. L. Lions, G. Papanicolaou, Asymptotic Analysis for Periodic Structures, North-Holland Publishing Company, New York, (1978).

[4] R. M. Bozorth, Ferromagnetism, van Nostrand, Toronto (1951).

[5] J. D. Carlson, D. M. Catanzarite and K. A. St Clair, Commercial magnetorheological fluid devices, Int. J. Mod. Phys. B 10 (1996), 2857-2865. 
[6] A. Defranceschi, An Introduction to Homogenization and G-convergence, Lecture Notes, School on Homogenization, ICTP, Trieste (Sept. 6-8, 1993).

[7] W. T. Doyle, The Clausius-Mossotti problem for cubic arrays of spheres, J. Appl. Phys. 49 (1978), 795-797.

[8] A. P. Gast and C. F. Zukoski, Electrorheological fluids as colloidal suspensions, Advances in Colloid and Interface Science 30, Elsevier Science Publishers, Amsterdam (1989), 153202.

[9] K. O. Havelka and J. W. Pialet, Electrorheological technology: the future is now, Chemtech 26 (1996), 36-45.

[10] V. V. Jikov, S. M. Kozlov and O. A. Oleinik, Homogenization of Differential Operators and Integral Functionals, Springer-Verlag, New York (1994).

[11] M. R. Jolly, J. D. Carlson and B. C. Muñoz, A model for the behavior magnetorheological materials, J. Smart Mater. 8 Struct. 5 (1996), 607-614.

[12] M. R. Jolly, J. D. Carlson, B. C. Muñoz and T. A. Bullions, The magnetoviscoelastic effect of elastomer composites consisting of ferrous particles embedded in a polymer matrix, J. Intel. Mater. Sys. Struct. 7 (1996), 613-622.

[13] T. B. Jones, Electromechanics of Particles, Cambridge University Press, New York (1995).

[14] J. B. Keller, Conductivity of a medium containing a dense array of perfectly conducting spheres or cylinders or nonconducting cylinders, J. Appl. Phys. 34 (1963), 991-993.

[15] J. Lam, Magnetic permeability of a simple cubic lattice of conducting magnetic spheres, J. Appl. Phys. 60 (1986), 4230-4235.

[16] J. C. Maxwell, A Treatise on Electricity and Magnetism, 3rd Ed., Dover Publications. New York (1892).

[17] Maxwell 3D Field Simulator, Ansoft Corporation, Pittsburgh, PA.

[18] B. K. P. Scaife, Principles of Dielectrics, Clarendon, Oxford (1989). 